\title{
Scolia
}

Revue de linguistique

$35 \mid 2021$

Superlatifs et définitude

\section{Pierre LE GOFFIC, Grammaire de la subordination en français}

\section{Claude Muller}

\section{OpenEdition}

\section{Journals}

Édition électronique

URL : https://journals.openedition.org/scolia/1535

DOI : $10.4000 /$ scolia. 1535

ISSN : 2677-4224

\section{Éditeur}

Presses universitaires de Strasbourg

\section{Édition imprimée}

Date de publication : 9 juillet 2021

Pagination : 173-187

ISBN : 979-10-344-0091-1

ISSN : 1253-9708

Référence électronique

Claude Muller, "Pierre LE GofFIc, Grammaire de la subordination en français », Scolia [En ligne], 35 | 2021, mis en ligne le 09 juillet 2021, consulté le 13 juillet 2021. URL : http://journals.openedition.org/scolia/ 1535 ; DOI : https://doi.org/10.4000/scolia.1535

Les contenus de la revue Scolia sont mis à disposition selon les termes de la Licence Creative Commons Attribution - Pas d'Utilisation Commerciale - Partage dans les Mêmes Conditions 4.0 International. 
Pierre LE GOFFIC, Grammaire de la subordination en français, Paris, Éditions Ophrys, 2019, 303 pages.

Ce livre paraît dans une collection totalement dédiée au français, spécialisée dans des présentations de synthèse sur des questions de langue et de linguistique. Les dix chapitres passent en revue, après une vue d'ensemble qui annonce le cadre et les principales articulations du livre, les différents types de subordonnées, en commençant par les interrogatives et exclamatives, suivies du chapitre sur les complétives. Viennent ensuite les «indéfinies nominale»: il faut y voir ce que l'on nomme ailleurs les relatives indépendantes ou libres. Les subordonnées "circonstancielles» regroupent toutes les constructions à conjonction complexe, celles qui équivalent à des compléments adverbiaux de cause, but, temps, etc. mais aussi les comparatives, les consécutives, les concessives. Les relatives ferment le ban, sachant qu'il s'agit dans ce cas des seules constructions à antécédent. La fin du livre est consacrée à des constructions plus particulières: un chapitre traite des clivées, dont la principale est toujours c'est; un autre examine les faits de «dépendance lointaine» des mots qu-. L'auteur inclut dans son étude les subordonnées à mode non fini, et termine par l'examen de cas de «dépendances textuelles non marquées».

Le livre tient ses promesses sur un point: l'exhaustivité. Le contenu examiné est très riche. En regard cependant, la bibliographie, sur un domaine aussi vaste, est assez maigrelette, mais elle ne sert pas à noter des renvois aux auteurs cités: presque aucun ne l'est. C'est une faiblesse, peut-être inévitable: le livre tend à affirmer un certain angle de vue, et ne s'embarrasse pas de discussions avec les théories alternatives. Le point de vue théorique est fixé assez sommairement dans les pages 13 à 15, comme faisant écho aux travaux de Sandfeld, Jespersen, Damourette et Pichon; il s'agit donc de faire sur cette question, de la grammaire «traditionnelle» (peut-on ranger Damourette et Pichon sous cette étiquette?) de façon ouverte et intelligente. On verra que sur certains points, le livre affirme une doctrine propre à l'auteur, qui n'est d'ailleurs pas nouvelle (on la trouve aussi dans la Grammaire de la phrase française (1993 pour sa première parution), ouvrage bien connu de Pierre Le Goffic.

Dans ce qui suit, je vais m'attacher à ce qui peut donner lieu à discussion, ce qui fatalement risque de donner une vision déséquilibrée 
à ce compte rendu: il me semble inutile de m'attarder sur la très grande part du contenu avec laquelle je suis en accord avec l'auteur. Mes remarques tiennent à mon propre point de vue sur ces questions, à partir de mes propres analyses, ainsi que de celles d'auteurs dont je partage les points de vue.

Un point central de l'exposé repose sur l'analyse catégorielle et syntaxique des classes de mots qu- qui forment en français (comme en italien, espagnol) le matériau de l'articulation entre les verbes subordonnés et leur terme recteur. Ce sont traditionnellement soit des relatifs-interrogatifs, soit des conjonctions, la ligne de partage étant que les pronoms entrent dans des relations de coréférence interne à la subordonnée, alors que les conjonctions ne le font pas. Généralement, on considère que le subordonnant le plus général et le moins "consistant» sémantiquement, le que des subordinations complétives, est sans fonction argumentale dans la proposition introduite, et forme la base de la catégorie des conjonctions de subordination. Pierre Le Goffic a choisi de décrire ce terme, conformément à son origine, comme un pronom indéfini neutre. Il refuse donc l'étiquette traditionnelle de conjonction de subordination, et rejette aussi la catégorie de mots que cette étiquette recouvre (p. 37: Les «conjonctions»: une catégorie superflue).

L'argumentation pour justifier de la nature pronominale de que se trouve p. 78: la complétive est une nominalisation; comment réaliser la transformation de la proposition en groupe nominal?

L'hypothèse la plus plausible (...) est que le que complétif n'est autre que le pronom indéfini neutre en qu- (non Hum), employé métalinguistiquement, avec ellipse de être, ainsi que le représente la glose : que P = quoi (ou ce que) P [est]. (p. 78).

Cette description repose cependant sur une assertion douteuse: celle que les complétives sont des groupes nominaux ${ }^{1}$. Ce qui est nominal, dans la description qu'on donne habituellement, c'est la position d'argument du complément du verbe principal pour les complétives compléments de verbe. L'hypothèse des complétives groupes nominaux, avancée par l'école guillaumienne, a été critiquée par eux-mêmes (voir par exemple Boone, 1994). En effet, de très

1 On peut noter aussi que dans cette définition que $P$ est considéré comme équivalent à ce que $P$, alors que les distributions ne sont pas les mêmes. 
nombreux contre-exemples montrent que les complétives en que peuvent être des équivalents d'adjectif (ici même, "complétives adjectives », p. 40 pour des emplois attributs), ou encore épithètes dans les constructions comme le fait que. Les constructions que $P$ ont aussi une vaste gamme d'emplois qui ne correspond pas à une distribution nominale, comme la complémentation d'un adverbe: heureusement que $P$, certainement que $P$, ou encore l'emploi isolé, impératif dans qu'il vienne!, ou hypothèse: qu'il vienne, et on est fichus. Et que faire aussi des subordinations «discursives» comme celle des incises populaires ${ }^{2}$ : $P$, qu’il me dit, où la principale «logique» est subordonnée?

La grammaticalisation du relatif indéfini latin ${ }^{3}$ dans les langues romanes a évidé totalement le contenu pronominal, pour ne laisser à ce que que sa fonction de subordonnant. Il serait donc plus juste de dire que les complétives en que sont aptes à entrer dans une position d'argument nominal complément de verbe, sans que cela en fasse des groupes nominaux.

La fonction première de que complétif est bien, comme le dit d'ailleurs Le Goffic «ce qui permet d'enchâsser P dans une structure supérieure $»^{4}$ : elle relève donc de la dépendance. Il n'est pas nécessaire pour ce faire que la tête de la complétive soit un pronom ou un ancien pronom, comme le montrent beaucoup de langues 5 . Cette description ne peut que réduire au statut d'exceptions ${ }^{6}$ toutes les constructions où les complétives n'entrent pas dans la distribution du groupe nominal. Cela dit, on peut accorder à Pierre Le Goffic non seulement que l'origine de que est pronominale, mais qu'il existe des complétives en

2 La construction est décrite p. 102 comme une structure obtenue par réduction d'un verbe est: «X» [est] (ce) qu'il m'a dit. Je ne pense pas que cette "glose» soit une véritable analyse, qui serait problématique puisque la forme résultante est nominalisée.

3 Il s'agit à l'origine d'une corrélation, dont le relatif indéfini était le corrélateur dans la subordonnée.

4 Page 78, par le biais d'une "transformation de 'P' en 'ce que $\mathrm{P}$ est'»

5 Il faut se rappeler que la conjonction complétive anglaise est facultative, mais il est vrai que le relatif direct à antécédent l'est aussi. Le breton, dans les langues indoeuropéennes, n'a pas de conjonction du type qu-pour ses complétives, pas plus qu'il n'a de pronom relatif. Il y a souvent un lien entre conjonction et relatif à antécédent (par ex. angl. that).

6 On retrouve cette question à de multiples endroits dans le livre: le que «béquille » du subjonctif; le que 'explétif de la complémentation adverbiale (p. 100); la structure «inanalysable» (p. 222) d'une clivée comme c'est demain que... 
ce que qui, elles, sont certainement nominales ${ }^{7}$ : dans ce cas, la structure est effectivement celle des relatives, avec une relation de coréférence globale entre le cataphorique ce et la complétive, au lieu d'une relation de pronom à argument interne. La logique du système défendu ici voudrait d'ailleurs que le que des complétives en ce que soit analysé comme un pronom relatif à coréférence globale, plutôt que comme un autre emploi du pronom indéfini neutre: le que des complétives ce que serait une forme fonctionnelle comme le que des relatives à antécédent, et non l'équivalent des pronoms des relatives sans antécédent (appelées ici 'subordonnées indéfinies nominales').

On sait que d'autres théories ont proposé des analyses assez différentes, comme la grammaire générative et ses variantes, avec une analyse des relatives à antécédent qui considère les formes fonctionnelles qui-sujet / que-non sujet comme des variantes de la conjonction que (hypothèse due essentiellement à Kayne, 1975), et l'idée essentielle que le début syntaxique de la sphère verbale est une position initiale, le complementizer ou "complémenteur» de son domaine, qui peut entre autres recevoir les pronoms interrogatifs et les pronoms relatifs, et qui est le point d'accueil possible d'une marque de la dépendance du verbe, matérialisée par que lorsqu'aucun autre morphème à fonction subordonnante ne s'y trouve. Est-ce encore un pronom? Finalement, la catégorie importe peu: le mot a pour fonction principale de marquer que le verbe fini $^{8}$ qui dépend de lui n'est pas autonome, soit parce qu'il dépend d'un terme recteur, soit parce qu'il ne reçoit pas de modalité énonciative9. Le terme conjonction peut gêner par sa connotation logiciste, mais quoi d'autre? "Enchâsseur» pour Wilmet (2003), "particule» pour Blanche-Benveniste (1990), ou pronom indéfini, comme ici, peu importe si on reconnaît d'une part l'évidement de la forme d'origine pronominale en fonction de conjonction, d'autre part son rôle de marqueur de dépendance, qui explique directement qu'on le trouve aussi bien dans les emplois isolés que dans les emplois en lien avec des recteurs de toute nature.

7 C'était pour Gross (1968: 99) la source des complétives en que, obtenues par «effacement» du ce; cela évitait d'avoir à décrire ces dernières comme des groupes nominaux.

8 Contrairement aux pronoms, le que complétif est totalement allergique à l'infinitif.

9 Il existe des que-P indépendants viables comme énoncés, mais leur interprétation énonciative reste vague, entre évocation, souhait ou ordre. 
Pourrait-on imaginer un continuum ? Wilmet $(2003, \$ 678)$ stigmatise les "arêtes vives» de la bipartition entre pronom et conjonction, et préfère imaginer une famille de fonctions que se répartissent les différents mots $q u$ - en français. On peut admettre dans ce cas, pour aller dans le sens de Pierre Le Goffic, un que complétif ayant encore des affinités avec sa famille d'origine, mais en contrepartie supposer que les relatifs, notamment les relatifs fonctionnels évoqués ci-dessus, aient des relations étroites avec la fonction de conjonction, puisque tous se trouvent dans la position de marqueur de dépendance. En somme, il y aurait deux extrêmes: d'un côté le que complétif, devenu pure marque de dépendance du verbe fini, vide de sens propre, mais apte à faire entrer la construction verbale dans une position d'argument direct, de l'autre les pronoms indéfinis à spécialisation («humain/non-humain»), et dans l'entre-deux les formes fonctionnelles non spécialisées, toutes aptes à marquer la dépendance, donc la subordination, et toutes plus ou moins réceptives à la coréférence avec une spécialisation fonctionnelle possible. Il reste que vouloir se passer, quel que soit le nom qu'on lui donne, de la catégorie de conjonction, qui fonctionne un peu comme celle de déterminant, en métacatégorie fonctionnelle de mots de provenance diverse ${ }^{10}$, oblitère un fait fondamental qui est qu'en français la dépendance du verbe conjugué soit à un recteur externe soit comme signe de non assertion utilise une position initiale de la construction argumentale du verbe, de telle façon qu'elle soit occupée par un terme à fonction subordonnante, dont le morphème non marqué est que.

Le chapitre II est consacré aux interrogatives et aux exclamatives. Le terme est préféré à « interrogative indirecte» de la tradition, qui renvoie à la notion de "discours indirect ${ }^{11}$ " pour signifier que la question n'est pas activée, mais rapportée à un autre locuteur ou une énonciation antérieure. Il me semble que le terme «interrogatives» peut se justifier effectivement parce que ces subordonnées ont des marqueurs

10 Je ne comprends pas l'objection qui est faite, parce que les termes utilisés dans la subordination sont souvent des pronoms ou des adverbes, que l'utilisation d'une autre catégorie serait inutile: les catégories peuvent comporter des ensembles englobants, comme les déterminants, déjà signalés, ou la négation; les proformes indéfinies relèvent elles aussi de catégories diverses, déterminants, pronoms, adverbes.

11 Et non pas à la complémentation « indirecte » (p. 49). 
(notamment pour des mots qu-comme quel, comment) identiques à ceux des questions, mais aussi parce que les relations sémantiques des deux types, indépendante et subordonnée, reposent sur une base commune, qui n'est cependant pas exactement l'interrogation. Plutôt que de garder une terminologie énonciative qui ne correspond pas à la réalité, puisqu'il s'agit de subordonnées, en parlant de "question virtuelle» (p.47), il me semblerait préférable de parler de subordonnées d'identification ${ }^{12}$; il n'y a pas de question cachée ou virtuelle dans je sais qui est venu / comment il a fait ça: il s'agit de sélectionner parmi des entités celle ou celles qui satisfont à un prédicat. C'est ce que fait aussi la question (directe), mais dans ce dernier cas, ce processus s'accompagne d'une démarche énonciative, la question, absente ici. Dans la subordonnée, cette sélection ou identification est signifiée sur une gamme de possibles, qui dans les interrogatives «totales» se limite à une pure alternative sur la vérité de l'action verbale: ou $P$, ou $\neg P$ (j'ignore s'il viendra) et dans les interrogatives "partielles» porte sur un argument: désignez qui a fait ça. C'est donc dans le premier cas, de la vérité d'une prédication enchâssée dont on parle, et dans le second cas, de la désignation d'un sous-ensemble qui satisfait à la prédication enchâssée. Dans le second cas, un mot mérite d'être mis à $\operatorname{part}^{13}$ : quel, marqueur de sélection (p. 56), sert en effet à expliciter tous les autres: combien = en quelle quantité, quand $=\dot{a}$ quel moment, qui=quelle personne, etc. Le rôle sémantique de la proforme interrogative est-il simplement de «représenter une variable indéfinie dans un domaine ontologique donné» (p. 51)? Je ne le crois pas. Un pur indéfini ${ }^{14}$, c'est une variable, effectivement, comme l'est le qui de qui que ce soit, n'importe qui. Dans le contexte particulier des «interrogatives", la variable est de plus membre d'une disjonction, avec la négation, sur la valeur de vérité de la relation qui l'associe à la prédication: à propos de $x$, $x$ est tel que $P(x)$, ou bien $x$ n'est pas tel que $P(x)$. C'est cette relation alternative qui constitue le socle commun des interrogatives totales et partielles $^{15}$ : dans l'interrogative partielle, l'ensemble de ces relations

12 Voir sur cette question Muller $(1996,2001)$. La dénomination compte cependant moins que le contenu qu'on donne aux catégories.

13 Il est effectivement signalé comme tel p. 58.

14 Voir sur les indéfinis Muller (2019).

15 Par exemple, Je sais qui est venu = je sais si x est venu (ou non), si y est venu (ou non), si z est venu (ou non). 
sur l'appropriation ou non de telle ou telle valeur de la variable est le véritable socle constitutif de l'emploi des mots interrogatifs, alors que l'indéfini se limite à être une pure liste de valeurs de la variable.

Le problème de la catégorie revient avec si (p. 50): faut-il abandonner dans ce cas la dénomination habituelle de conjonction pour parler $\mathrm{d}^{\prime}$ "adverbe de phrase " ${ }^{16}$ ? On perd dans ce cas tout lien avec le rôle subordonnant de $s i$, qui est tout de même plus proche du $s i$ hypothétique que du si des mots-phrases. Ici aussi, l'idée implicite que la catégorie est unique et non hybridable est excessive: les si de la subordination ont à la fois la fonction englobante de «subordonnant» et celle équivalent à un complexe morphologique qui, dans le cas des hypothétiques, serait à peu près au cas où, locution adverbiale centrée sur la «localisation» dans le domaine du vrai. Le si des interrogatives serait plutôt centré sur l'identification d'une valeur dans une alternative, un peu comme quel cas c'est de $P$ ou $\neg P^{17}$.

La structure "supplétive» (p. 53) avec ce est bien décrite, comme relevant de la syntaxe des relatives. Cependant, ici aussi, le contenu n'est pas exactement le même. La discussion sur la "perspective percontative» amorce la réponse, mais en reste là ${ }^{18}$ :

${ }^{*}$ Ce qui ne va pas? nest pas en soi une phrase interrogative, il faut $y$ ajouter un mot en qu- (Qu'est... ?) pour former Qu'est-ce qui ne va pas? (p. 65).

Le verbe être est central dans les interrogatives, parce qu'il établit la relation entre l'indéfini qui entre dans la position argumentale, et les propriétés de celui-ci: l'indéfini cible de la question est toujours attribut caché de cette relation: ce qui ne va pas est quoi? Dans la subordonnée interrogative, on a toujours une forme longue paraphrasant la subordonnée, qui met en évidence cette relation attributive: Je sais qui est venu = Je sais quelle est / quelles sont la/les personnes qui est/

16 Dans le cas des interrogatives à l'infinitif (p. 61), comment distinguer, si l'introducteur est simplement qualifié d'adverbe sans préciser de fonction subordonnante, je sais où aller (interrogative) et je veux y aller (complétive, avec un locatif $y$ en tête aussi)? C'est l'occupation par où de la position de subordonnant qui fait la différence.

17 D'où dans certaines langues, des mots $q u$ - pour cette alternative, comme whether en anglais. Il est assez difficile de paraphraser simplement le contenu de l'interrogative totale, mais le noyau de la subordonnée et la cible de la position d'argument du verbe principal est la relation à ce qui est ou non «le cas» à propos de $\mathrm{P}$.

18 La même amorce de réponse figure p. 115-116 pour les formes 'renforcées' des questions. 
sont venue(s). Il en va de même avec ce que: J'ignore ce qu'il fait = j'ignore quelles sont les choses qu'il fait. La relative n'a jamais cette expansion: J'apporte ce qu'il a fait ne peut signifier ${ }^{\star} J$ 'apporte quelles sont les choses qu'il a faites. Il me semble que cette propriété, cruciale, devrait être signalée pour montrer que les indéfinis des interrogatives se distinguent sémantiquement et fonctionnellement des indéfinis prototypiques $^{19}$.

La description des exclamatives présente un panorama très complet de ces subordonnées souvent négligées. La frontière entre les exclamatives à marqueur indéfini-interrogatif et les interrogatives est assez mince, et l'emphase ne suffit pas à les caractériser, comme le montre l'exemple $T u$ vois où tu es allé te fourrer! (p. 68) qui est en effet comme le dit l'auteur une interrogative. Dans les constructions à marqueur spécifique, le si est un adverbe de degré, en plus de sa fonction de subordonnant (je ne comprends pas la remarque «Si porte sur l'ensemble de la proposition, et n'a pas de fonction identifiable» (p. 68), à propos de Vous savez si je vous aime! : c'est un adverbial de degré «à quel point», comme il est d'ailleurs glosé dans la suite). Sur la sémantique des exclamatives, je ne partage pas ce qui est dit p. 75-76: «on peut ranger les subordonnées exclamatives avec les interrogatives, malgré leurs différences, dans la perspective percontative». Il me semble qu'il y a une différence essentielle: il n'y a absolument pas, dans l'exclamative, qu'elle soit indépendante ou subordonnée, de sélection de valeurs de la variable selon le vrai et le faux. Je me range à l'avis de Milner (1978: 260): l'exclamative, au contraire de l'interrogative, «ne suppose aucune incertitude ${ }^{20}$ concernant l'élément précédé du marqueur qu-». Il explique, avec l'exemple Je sais à quel point vous l'aimez!, que le marqueur de degré variable, à tel ou tel point, est toujours validé dans l'exclamation ${ }^{21}$, d'où le sens de haut degré sur le

19 De même que certains noms, pour compléter ce qui est dit à ce propos, p. 66. Le concept d'heure n'est pas le même dans je savoure l'heure de sa venue et je sais l'heure de sa venue.

20 Il s'agit de la sélection de valeurs de la variable qui satisfont la prédication dans le cas des interrogatives: il y a toujours la possibilité que certaines valeurs ne la permettent pas.

21 Suivant Milner, la relation vous l'aimez à tel point est assertable pour toute valeur jusqu'à un maximum (ce sommet de l'échelle d'intensité justifie l'exclamation), d'où le haut degré des exclamatives. La sémantique diffère donc assez nettement. Pour les exclamatives, il y a sans doute des limites pragmatiques à l'échelle des degrés associée 
prédicat: il n'y a pas d'incertitude, dans l'exclamative indirecte, sur les valeurs de l'échelle d'intensité associée, quant à la validité de l'assertion indirecte dans la subordonnée.

Le chapitre III développe l'analyse des complétives comme des subordonnées à tête indéfinie, avec une certaine retenue ( $q u e$ 'connecteur pur' en apparence, 'conjonction' si on veut», (p. 79)). Ce qui est dit des constructions complétives en ce que mériterait une explication plus approfondie: est-ce que ce que est vraiment une "locution équivalenteau que complétif» (p. 79)? L'analyse est exacte, qui fait de que dans cette locution «un pronom relatif en sous-jacence» (p. 79), avec une coréférence globale à la subordonnée. Mais si on en tire les conséquences, le que $P$ des complétives ce que $P$ ne peut être un groupe nominal, c'est une épithète fonctionnelle; et le rapprochement de cette "relative» à coréférence globale, qui ressemble beaucoup à la conjonction, avec les relatives en que à coréférence interne ne paraît plus aberrant. Surtout, cela invite à se poser la question suivante: les complétives en que pourraient être des formes défectives, tronquées, donc sous-déterminées. Cela expliquerait leur polyvalence fonctionnelle, non seulement en termes de catégorie, mais aussi en termes de fonction, puisque de nombreuses complétives de type Prép-ce-que $P$ ont un équivalent que $P$ (se souvenir que $P$, veiller que $P$ ). Enfin, en termes d'analyse syntaxique fine, il me semble indispensable d'analyser ce que puisque le ce est dans la principale en tant qu'antécédent, alors que que est au début de la subordonnée ${ }^{22}$. Toujours dans les complétives à tête nominale, il ne me semble jamais justifié d'analyser le fait que $P$ comme une structure à complément de nom qui serait *le fait de ce que $P$ (p. 93): les contenus sémantiques du nom et du pronom sont les mêmes.

Dans les emplois particuliers, je relève (p. 98-99) les types 2 et 3 exemplifiés par Qu'est-ce que c'est que l'amour? et Triste chose que la solitude! C'est typiquement le genre de questions qu'on préfère glisser sous le tapis, parce qu'elles dérangent. Le premier type est-il vraiment la réduction par ellipse d'une complétive en être? C'est assez

au prédicat, ce qui tempère un peu la présentation sommaire faite ici (voir Muller 1996: 234).

22 L'argument du figement ou même $\mathrm{du}$ "mot» unique pour ce que ne vaut pas plus, pour l'analyse syntaxique, que celui du mot unique au pour refuser d'analyser la suite $\grave{a}+l e$. 
peu convaincant, et encore moins pour le type suivant. Le Goffic suit ici l'opinion de Damourette et Pichon, qui parlent d'ellipse ( $\$ 3055)$ mais est-ce convaincant quand la phrase non ellipsée est à peu près inacceptable: ${ }^{*}$ u'est-ce que c'est que l'amour est ${ }^{23}$ ? Même si c'est le $\operatorname{cas}^{24}$, il faudrait en parler dans les constructions de type relatif, à coréférence interne à la subordonnée (le pronom coréfère à l'attribut absent du verbe ellipsé), et pas dans le chapitre des complétives.

Dans le chapitre IV, je ne trouve pas totalement légitime de classer sous la rubrique "subordonnées indéfinies nominales» les constructions appelées ici "relatives substantivées», ce que dit Marie est juste (p. 104). Il s'agit de relatives à antécédent, et le démonstratif $c e$ est sans doute sémantiquement vague, mais grammaticalement tout à fait défini ${ }^{25}$ ! D'autre part, aligner les constructions en ce sur les constructions en qui (parfois quoi), pronoms à contenu sémantique différencié, est assez trompeur pour l'analyse syntaxique:malgré le figement et la cliticisation de $c e$, ou l'absence d'autonomie de celui, ces termes sont dans la principale, alors que le relatif indépendant représente seul les contenus nominaux dans les deux propositions. Inversement, je ne comprends pas non plus pourquoi les constructions à adverbe relatif, où, quand, sont placées dans le chapitre suivant: certes elles ne sont pas à tête nominale, mais on peut minimiser la différence fonctionnelle pour voir en elles aussi des constructions à "chevillage» comme le dit Le Goffic en reprenant l'expression de Damourette et Pichon, ou dans une terminologie plus courante, des relatives indépendantes ${ }^{26}$.

Le chapitre $\mathrm{V}$ regroupe donc deux ensembles: d'une part les "subordonnées indéfinies adverbiales", soit toutes celles qui sont basées sur des adverbes en $q u$-, liste qui inclut ici le si hypothétique, d'autre part les subordonnées introduites par des «locutions conjonctives».

23 Un peu moins avec inversion:?qu'est-ce qu'est l'amour?

24 J'ai suggéré (Muller, 2013) un développement récent de que comme introducteur de rappel de thème sous certaines conditions, ce qui permettrait d'éviter l'analyse par ellipse. Cela reste problématique.

25 Il s'agit bien entendu de relatives restrictives, et l'introducteur démonstratif, que ce soit celui ou ce, équivaut à un groupe nominal défini (voir pour celui Hirschbühler \& Labelle, 1990; pour ce Muller, 2020).

26 C’est généralement admis pour où, mais il me semble aussi que quand est l'équivalent, pour un argument «temps», du qui «humain» pour les personnes dans les relatives indépendantes. 
On retrouve donc, indirectement, la catégorie «conjonction» écartée au début du livre. Le classement sémantique (tableau, p.125) distingue circonstances, qualité/quantité, et relations logiques. On notera qu'avant que est une locution conjonctive de temps (pour moi, une comparative sur le temps). Les «relations logiques» devraient être analysées comme des prédications dont l'argument complément est une complétive, ce qui les distinguerait des constructions à marqueur de degré (du type relatif). C'est ce que propose l'analyse qui est faite p. 128-129, mais avec une incertitude sur le «connecteur $q u$ - » : s'agit-il toujours du pronom indéfini, ou d'une simple marque d'enchâssement du verbe qui suit, et qui est donc le véritable argument du prédicat qu'est le début de la locution conjonctive? Les exemples d'analyses montrent qu'effectivement on trouve selon les cas un relatif (au moment où) et dans d'autres cas (pour que) le connecteur des complétives, qui doit donc être l'indéfini neutre. La difficulté de l'analyse, due au figement des conjonctions complexes, est rapportée p. 130. Selon Le Goffic,

Les dites «subordonnées circonstancielles introduites par des locutions conjonctives" sont simplement des Groupes Prépositionnels ou des Groupes Adverbiaux, à l'intérieur desquels se trouve enchâssée une subordonnée (complétive, relative, ou comparative) (p. 130-131).

Mais l'auteur corrige cette approche, obligé de prendre en compte la spécificité de locutions comme parce que ou tandis que, uniquement utilisables comme introducteurs de subordonnées, non classables comme adverbes ou prépositions. La «locution conjonctive» reste donc utilisée en cas de figement.

Sur l'analyse des différents types, je note, à propos des comparatives, la reconnaissance d'un que spécifique, adverbe indéfini de quantité ou de degré (p. 159). En revanche, hormis le signalement d'un "ne dit explétif» (p. 163), on ne trouve pas d'analyse détaillée de la comparative d'inégalité (on en reste à la tripartition classique, (p. 161), supériorité, infériorité, égalité, alors que la bipartition entre égalité et inégalité est première, marquée comme telle par exemple en anglais, allemand, par une différence de connecteur). La difficulté d'établir une corrélation 
(qui repose toujours sur des marqueurs co-indiciés) dans l'inégalité n'est pas signalée ${ }^{27}$.

Dans l'analyse des consécutives, (p. 171), se pose la question du lien entre le degré exprimé en principale: Paul était si fatigué et le fait décrit en subordonnée: ...qu'il s'est endormi. Les consécutives ne présentent pas de signe d'une corrélation effective, elles ont l'allure de complétives, et dans de nombreuses langues, elles sont introduites non par un pronom comparatif, mais par la conjonction. Je ne pense pas que l'explication donnée (p. 171) pour cet exercice est si difficile qu'on ne peut pas le faire corresponde à une paraphrase: «le degré de difficulté de cet exercice est égal à: on ne peut pas le faire». Le marqueur si n'est pas un corrélateur strict, mais un indice imprécis de haut degré, différent en cela du aussi. De plus, de nombreuses consécutives affirmatives ${ }^{28}$ sont marquées par une rupture énonciative. Le type de marqueur et cette rupture énonciative permettent de supposer, comme d'autres auteurs l'ont fait ${ }^{29}$, que ce qui distingue la consécutive, c'est qu'elle n'est pas en lien de coréférence directe par un marqueur de degré avec sa principale, mais qu'elle signale plutôt une conséquence, parmi d'autres, d'une zone de haut degré qui la rend possible.

Un mot sur les concessives: p. 188, il est question de ce qu'on appelle ailleurs les concessives extensionnelles, celles qui sont formées d'un indéfini, qui peut être les simples qui, quoi ou des formes augmentées, n'importe qui / quoi, qui / quoi que ce soit dans quoi qu'on fasse, il n'est jamais content. Je ne pense pas qu'il faille chercher, ici comme ailleurs, de "réduction de structure tautologiquefaites quoi que vous fassiez» (p. 188) (pour quoi que vous fassiez). Le que de ces concessives est à chercher dans une coordination d'éventualités, c'est donc le que des indépendantes qui signifie ici l'éventuel: qu'on fasse $x, y, z \ldots$ etc $^{30}$. L'indéfini (ici quoi, mais c'est valable pour ses variantes augmentées)

27 Voir Muller (1983, 1996). La présence d'une négation en subordonnée y trouve son explication, ainsi que son caractère explétif. Ce qui n'est pas du tout reconnu ici, c'est le caractère variable du pôle de référence dans les comparatives (alors que la corrélation est bien reconnue, p. 159).

28 La négation modifie nettement les consécutives: Cet exercice n'est pas si difficile qu'on ne puisse le faire.

29 Voir Allaire (1977), Milner (1978), et mon chapitre sur les consécutives (Muller, 1996).

30 Il y a une ressemblance avec l'interrogation, en ce que la structure la plus basique de la concession envisage un fait et sa négation: qu'il pleuve ou non, je sortirai. 
est en réalité une somme disjonctive des valeurs de la variable, couvrant toute l'étendue des suppositions possibles. L'indéfini est bien régi par le verbe, en effet, mais est antéposé sur le modèle des clivées, par focalisation, et il doit laisser dans la conjonction que un relais de sa fonction, permettant, comme dans les clivées, l'alternance entre qui et que: Quoi que ce soit qui arrive, c'est toujours de notre faute. Il faut donc supposer, dans cette hypothèse, qu'il y ait interchangeabilité entre le que complétif et le que relatif, exactement comme dans les clivées.

Un mot pour terminer cette revue de questions, à propos des clivées. Dans le cas des constructions à préposition, les trois variantes sont bien signalées: c'est vous à qui je parle, c'est à vous à qui je parle, c'est à vous que je parle(p. 227). Le troisième est signalé comme «tour inanalysable», parce que la fonction indirecte est marquée sur l'antécédent, et n'apparaît pas au niveau du que. L'analyse se basant sur un que conjonction et support possible de marques fonctionnelles décrit au contraire très naturellement ces différentes constructions ${ }^{31}$. C'est même un argument fort pour considérer l'interchangeabilité dans que de l'occurrence conjonctionnelle (pas de fonction marquée) avec l'occurrence pronominale (marquée fonctionnellement) ${ }^{32}$. La solution d'une marque polyvalente que support selon les cas, de fonctions d'arguments coréférents ou non et marqueur de dépendance verbale évite ce cul de sac de l'analyse grammaticale. Il en va de même pour les autres tours jugés ici inanalysables ou 'gallicismes' de la p. 228 comme c'est demain qu'on aura les résultats.

Il est clair que dans l'analyse développée ici, les dépendances lointaines sont difficiles à expliquer. Par exemple, pour Qui crois-tu qui va gagner? (par antéposition du qui à partir de Tu crois que qui va gagner?) la solution qui permet de marquer sur la conjonction de la complétive la fonction sujet obligatoirement signifiée est simple et élégante, permise simplement par l'antéposition du qui interrogatif

L'introduction d'une variable indéfinie construit de façon similaire un enchaînement de disjonctions.

31 Voir Muller (2003) sur la syntaxe et l'origine historique des trois constructions.

32 Conjuguée à l'obligation de marquer la fonction sujet dans sa proposition, cela nécessite d'avoir: c'est Jean qui conduit vs ...*que conduit. On notera ici que la subordonnée n'est absolument pas analysable sémantiquement comme une relative. 
et par la marque de coréférence fonctionnelle laissée sur $q u e^{33}$. Le Goffic doit supposer une source inacceptable, qui est ${ }^{*} T u$ crois qui qui va gagner?, avec un notable changement de structure, puisque, sans déplacement du pronom interrogatif, on a affaire à une complétive, alors que la modification suggérée aboutit à une relative peu crédible par ailleurs. Je ne pense pas que les autres solutions suggérées (évoquées p. 245) soient "plus compliquées et plus destructrices pour l'économie d'ensemble du système des mots $q u$-». De plus, cette solution (qui simplifierait grandement aussi l'analyse des clivées «inanalysables» et autres gallicismes), faut-il le dire, irait dans le sens de son hypothèse principale: si la conjonction que est de façon plus ou moins cachée un pronom neutre, pourquoi ne pourrait-elle pas transmettre les relations de coréférence et servir de support fonctionnel dans les structures de type relatif?

Je ne peux faire autrement que marquer mes principaux désaccords avec certaines des analyses de ce livre. Pourtant, il contient quantité de faits, des données riches, des analyses ingénieuses. Il n'en est que plus regrettable qu'il soit aussi fermé aux analyses venant de théories concurrentes à la sienne, jamais discutées. Le livre se contente trop souvent de signaler des problèmes, présentés comme inanalysables, ou, comme c'est trop souvent le cas en grammaire, comme faisant exception à la règle. Il sera certainement une somme utile aux étudiants et aux grammairiens, c'est le point le plus important, et sans doute le but visé par son auteur.

Claude MULLER

Université Bordeaux Montaigne \& CNRS

claude.muller31@orange.fr

\section{Références}

ALLAIRE S. (1982 [1977]), Le Modèle syntaxique des systèmes corrélatifs, thèse de doctorat d'Etat, Rennes-2, Presses Universitaires de Lille-III, Paris, Champion, 1982.

BLANCHE-BENVENISTE C. (1990), Usages normatifs et non normatifs dans les relatives en français, en espagnol et en

33 Historiquement, ce qui pourrait avoir été grandement facilité par la prononciation [i] en français classique du il (personnel ou impersonnel), d'où des qu'il prononcés qui. 
portugais, in Bechert J. et al. (éds), Towards a Typology of European Languages, Berlin-New York, Mouton de Gruyter, 317-355.

BOONE A. (1994), La complétive: un cas de nominalisation externe? Travaux de linguistique 27, 29-42.

DAMOURETTE J. \& PICHON E. (1911-1940), Des mots à la pensée, Essai de grammaire de la langue française, Paris, D’Artrey.

GROSS M. (1968), Grammaire transformationnelle du français, Syntaxe $d u$ verbe, Paris, Larousse.

HIRSCHBÜHLER P. \& LABELLE M. (1990), Celui comme noyau de syntagme nominal, Travaux de linguistique 20, 109-122.

KAYNE R.S. (1975), French Relative Que, Recherches linguistiques de Vincennes, 2 (40-61) et 3 (27-92); aussi (1976), in Hensey F. \& Luján M. (éds), Current Studies in Romance Linguistics, Washington D.C., Georgetown University Press, 255-299.

LE GOFFIC P. (2005) [1993], Grammaire de la phrase française, Paris, Hachette.

MILNER J.C. (1978), De la syntaxe à l'interprétation, Paris, Le Seuil.

MULLER C. (1983), Les comparatives du français et la négation, Linguisticae Investigationes VII : 2, 335-362.

MULLER C. (1996), La subordination en français, Paris, Armand Colin.

MULLER C. (2001), Sémantique de la subordination: l'interrogation indirecte, in Rousseau A. (éd.), La sémantique des relations, Lille, Editions de Conseil Scientifique de l'Université Charlesde-Gaulle - Lille 3, 163-177.

MULLER C. (2003), Naissance et évolution des constructions clivées en "c'est...que...»: de la focalisation sur l'objet concret à la focalisation fonctionnelle, in Blumenthal P. \& Tyvaert J.E. (éds), La cognition dans le temps, Tübingen, Niemeyer, 101-120.

MULLER C. (2013), Que entre conjonction et pronom clitique en français, in Jacob D. \& Ploog K. (éds), Autour de que - El entorno de que, Studia Romanica et linguistica, Frankfurt am Main, Peter Lang, 113-138.

MULLER C. (2019), Indéfinis et partitifs en français, Bordeaux, Presses Universitaires de Bordeaux.

MULLER C. (2020), Le ce antécédent des constructions de type relatif: un pronom démonstratif?, Langue française 205, 101-119.

WILMET M. (2003), Grammaire critique du français, Bruxelles, Duculot (3e édition). 